tion gar nicht so schlecht ausgestaltet ist. Die Länder konnten nämlich im Zuge der Europäisierung zahlreiche Konzessionen erreichen, so in Bezug auf Informationsrechte und das Recht, Stellungnahmen zu veröffentlichen. Realiter hat aber vor allem der Landeshauptmann gewonnen, also die Exekutive.

Die Güte eines Sammelbandes steht und fällt nicht nur mit der Qualität der Einzelbeiträge, sondern hängt maßgeblich auch von der thematischen und theoretischen Einordnung ab. In dieser Hinsicht kann das Werk nicht überzeugen, da ein gemeinsamer Horizont nicht skizziert und Thesen wie Themen nicht zusammengebracht werden. Natürlich kann der Leser wiederkehrende Annahmen finden, die in unterschiedlichen Kontexten bestätigt oder angezweifelt werden. Den Rahmen dafür muss er sich aber selbst schaffen. Lässt er sich darauf ein, wird er mit einzelnen guten Beiträgen belohnt. So warten Leuprecht, Hazell / Paun und auch Watts mit interessanten Argumentationen auf, während andere Autoren von einer These geleitete Einblicke in Entwicklungslinien einer Institution geben, wie dies Kincaid für den US-Senat unternimmt. Andere Texte verharren hingegen in der Deskription.

Es sind also Probleme zu konstatieren, die für Sammelbände, die aufTagungen beruhen, typisch geworden sind. Die Einzeltexte stehen monolithisch nebeneinander, ihre Qualität variiert, sie treten in keinen Dialog miteinander und finden sich erst nach mehreren Jahren zwischen zwei Buchdeckeln wieder, ohne aktualisiert worden zu sein. So geben die Beiträge hier Auskunft über den Stand der Diskussionen von 2006.

Dennoch ist es aufgrund seiner interessanten Informationen und Details ein gutes, ergänzendes Buch. Man findet hier keine bahnbrechenden Neuigkeiten oder überraschenden Thesen, aber man kann Einsichten gewinnen, so zum Beispiel zum Forschungsfeld der interparlamentarischen Kooperation, das in Zukunft vermutlich wachsende Aufmerksamkeit erfahren wird.

Franziska Höpcke

\title{
Parlamente als Wahlorgane: theoretisch fundiert und empirisch facettenreich
}

Sieberer, Ulrich: Parlamente als Wablorgane. Parlamentarische Wablbefugnisse und ihre Nutzung in 25 europäischen Demokratien (Studien zum Parlamentarismus, Band 14), Nomos Verlagsgesellschaft, Baden-Baden 2010, 324 Seiten, € 39,-.

Die vergleichende Parlamentsforschung hat seit den 1990er Jahren große Fortschritte gemacht. Diesen Fortschritten zum Trotz konstatiert Ulrich Sieberer mit Recht eine große Forschungslücke: Die Rolle von Parlamenten als Wahlgremien für Regierungen wie für eine Reihe weiterer öffentlicher Ämter wurde bislang nicht eingehend untersucht. Sieberer wirft daher folgende Fragen auf (S. 29 - 30): Welche Erwartungen ergeben sich theoretisch aus parlamentarischen Wahlbefugnissen? Welche Wahlbefugnisse von Parlamenten können in den untersuchten europäischen Demokratien beobachtet werden? Welche Faktoren bestimmen das Wahlverhalten der Parteien in diesen Parlamenten? Zudem: Welche Eigenschaften haben die von den Parlamenten gewählten Amtsinhaber? Diese Fragen werden für Exeku- 
tivämter (Premier- und Fachminister) sowie für Ämter in den von Sieberer so genannten Schrankeninstitutionen (Staatsoberhaupt, Zentralbankpräsidentschaft, Rechnungshofpräsidentschaft, Ombudsstellen und Verfassungsgerichte) bearbeitet. Untersucht werden alle EU-Länder außer Rumänien und Bulgarien.

Das Buch gliedert sich in drei Teile. Im ersten wird die Wahlfunktion von Parlamenten konzeptionell diskutiert und sodann die institutionelle Ausgestaltung der Wahlbefugnisse analysiert. Der dritte Abschnitt ist einer detaillierteren Analyse des Wahlverhaltens der wählenden Akteure gewidmet.

Konzeptionell geht die Arbeit von delegationstheoretischen Vorstellungen aus. Repräsentative Demokratien werden im Sinne von Kaare Strøm als Delegationskette aufgefasst ${ }^{1}$ und zwei ihrer Glieder erforscht: die Delegation vom Parlament an exekutive Akteure und die Übertragung von Kontrollbefugnissen an Schrankeninstitutionen. Mit Bezug auf die Wahlen von Regierungsakteuren wird die Erwartung formuliert, dass die Wahlmöglichkeiten im positiven Parlamentarismus (Wahl der Exekutive) und im negativen Parlamentarismus (Abwahl der Exekutive) nicht ergänzende, sondern substitutive Kontrollmöglichkeiten des Prinzipals Parlament gegenüber dem Agenten Exekutive sind (S. 43).

Mit Blick auf die Besetzung von Ämtern in Schrankeninstitutionen konstatiert Sieberer, dass diese bislang mit Ausnahme der Verfassungsgerichte kaum Gegenstand vergleichender Analysen waren. Daher stellt er zunächst dar, an welche Voraussetzungen die Fähigkeit dieser Institutionen, restringierend tätig zu werden, gebunden ist (institutionelle Ressourcen und Anreize zu ihrem Einsatz). Den ersten Teil abschließend entwickelt der Autor drei Indizes zur Messung der institutionellen (Wahl-)Macht von Parlamenten (S. 74 ff.). Dabei geht er sehr überzeugend und hervorragend dokumentierend mit allfälligen Problemen von Indexbildungen in der vergleichenden Forschung um.

Der zweite Abschnitt des Buches steht im Zeichen der vergleichenden Empirie. Zunächst schildert der Autor seine Vorgehensweise und die Datenquellen. Diese Darstellungen werden durch einen ausführlichen Anhang unterstützt. Einer Deskription der in den Untersuchungsländern vorgefundenen Situation, die allein schon hochgradig informativ und lesenswert ist, folgen Analysen struktureller Zusammenhänge. In Bestätigung seiner oben zitierten Erwartung findet Sieberer, dass Parlamente Delegationsverluste durch Exekutivagenten tatsächlich entweder ex post oder (!) a priori zu vermeiden versuchen. Diese Kontrollmodi sind also vorrangig substitutiv, nicht ergänzend angelegt. Dasselbe gilt allerdings nicht für die Schrankeninstitutionen. Das liegt vor allem daran, dass deren Besetzungsregeln, die ja Unabhängigkeit sichern sollen, kaum nachträgliche Kontrollen zulassen. Ex-ante-Mechanismen zur Vermeidung von Delegationsverlusten haben hier also notwendigerweise Vorrang. Der Autor schließt daraus auf die Notwendigkeit, bei empirischen Arbeiten grundsätzlich beide Dimensionen zu erfassen.

Im dritten Teil des Werkes untersucht Sieberer reale Wahlprozesse zur Besetzung von Ämtern in Schrankeninstitutionen. Als Voraussetzung dafür beschreibt er eine komplexe Nutzenfunktion der wählenden Parteien. Diese hat eine klassische räumliche (ideologische Distanz) und zwei nichträumliche Komponenten. Deren erste, die Eignung des zu Wählenden für das Amt, geht ebenso auf ein Modell von James M. Enelow und Melvin J. Hinich

1 Vgl. Kaare Strøm / Wolfgang C. Müller / Torbjørn Bergman (Hrsg.), Delegation and Accountability in Parliamentary Democracies, Oxford 2003. 
aus dem Jahre 1982 zurück wie die räumliche Komponente. ${ }^{2}$ Mit der zweiten nichträumlichen Komponente modelliert Sieberer explizit, dass nicht nur Eigenschaften von Kandidaten, sondern etwa politische Rücksichten Einfluss auf Wahlentscheidungen haben können. Schließlich wird in dem Modell der Tatsache Rechnung getragen, dass unterschiedliche Akteure den einzelnen Komponenten der Nutzenfunktion unterschiedliches Gewicht beimessen. Die aus dem Modell abgeleiteten Hypothesen bestätigen sich mit Blick auf die erwarteten Effekte der räumlichen und der ersten nichträumlichen Komponente. Wettbewerbskontext und institutionelle Variablen erweisen sich dagegen bereits bei der Ableitung eindeutiger Hypothesen als schwierig. Die empirische Überprüfung bringt ebenfalls keine eindeutigen Resultate hervor.

Folgende Untersuchungsergebnisse werden im abschließenden Kapitel festgehalten: (1) Parlamente sind nicht „nur“ als Gesetzgebungsorgan, sondern auch - als durch Wahlen Macht verteilende Organe - relevante politische Institutionen. Dass diese Wahlen nachweisbar nicht reine Konsens- oder Zustimmungsmechanismen sind, sondern Ereignisse mit offenem Ausgang, rechtfertigt allemal ihre eingehende empirische Analyse. (2) Die delegationstheoretische Perspektive erweist sich als theoretisch fruchtbar und als empirisch tragfähig, denn die untersuchten Wahlergebnisse können aus räumlichen und nichträumlichen Modellkomponenten heraus gut erklärt werden.

Sieberers Schrift ist als Dissertation bei Wolfgang C. Müller in Mannheim entstanden, und es ist lobend festzustellen, dass man ihr in Stil, Vorgehensweise und Qualität anmerkt, aus welcher Schule sie kommt. Das Werk ist ein weiterer Nachweis der konzeptionellen Schärfe wie auch der empirischen Leistungsfähigkeit, die die vergleichende Parlamentsforschung in den letzten zwei Jahrzehnten erreicht hat. Dabei präsentiert der Autor komplexe theoretische Modelle und empirische Analysen so, dass auch dem technisch weniger versierten Leser immer wieder das Aufgreifen des „Handlungs“fadens ermöglicht wird. Gleichzeitig ist das Buch eine sehr verdienstvolle Datensammlung, die bisherige empfindliche Informationslücken für die vergleichende Parlamentsforschung schließt.

Natürlich gibt es auch Mängel. Die Messung der Idealpunkte der Personen, die Ämter in Schrankeninstitutionen besetzen, erscheint nur bedingt verlässlich. Mitunter werden recht weitreichende Schlussfolgerungen auf der Basis einer schmalen Datenlage gezogen. Wollte man diesen Kritikpunkten aber praktisch begegnen, müsste man eine andere Vorgehensweise wählen und ein anderes Buch schreiben. Das räumt Sieberer auch freimütig ein, wenn er darauf verweist, dass an verschiedenen Stellen Fallanalysen sinnvoll oder sogar wünschenswert wären, um Befunde zu vertiefen. Diese Kritik nimmt der Arbeit aber nicht ihren Wert, eher im Gegenteil. Vertiefende Fallanalysen bedürfen immer wieder der vergleichenden Rückbindungsmöglichkeit. Und dafür leistet die vorgelegte Publikation theoretisch, analytisch und in der deskriptiven Bereitstellung von Informationen Hervorragendes.

Kai-Uwe Schnapp

2 Vgl. James M. Enelow / Melvin J. Hinich, The Spatial Theory of Voting: An Introduction, Cambridge 1984 . 